\title{
Surface Topography of Microtubule Walls Decorated with Monomeric and Dimeric Kinesin Constructs
}

\author{
Andreas Hoenger ${ }^{1}, *$, Monika Doerhoefer ${ }^{1}$, \\ Guenther Woehlke', Peter Tittmann ${ }^{3}$, \\ Heinz Gross ${ }^{3}$, Young Hwa Song ${ }^{4}$ and \\ Eckhard Mandelkow ${ }^{4}$ \\ ${ }^{1}$ European Molecular Biology Laboratory, Meyerhofstr. 1, \\ D-69012 Heidelberg, Germany \\ ${ }^{2}$ Ludwig-Maximilians University, Adolf Butenandt \\ Institute of Cell Biology, Schillerstr. 42, \\ D-80336 Munich, Germany \\ ${ }^{3}$ Institute of Applied P hysics, S wiss Federal Institute \\ of Technology, Hoenggerberg, $\mathrm{CH}-8093$ Zürich, \\ Switzerland \\ ${ }^{4}$ Max-Planck-Unit for Structural Molecular Biology, \\ DESY-Hamburg, Notkestr. 85, \\ D-22603 Hamburg, Germany \\ *Corresponding author
}

The surface topography of opened-up microtubule walls (sheets) decorated with monomeric and dimeric kinesin motor domains was investigated by freezedrying and unidirectional metal shadowing. Electron microscopy of surface-shadowed specimens produces images with a high signal/noise ratio, which enable a direct observation of surface features below $\mathbf{2} \mathbf{~ n m}$ detail. Here we investigate the inner and outer surface of microtubules and tubulin sheets with and without decoration by kinesin motor domains. Tubulin sheets are flattened walls of microtubules, keeping lateral protofilament contacts intact. Surface shadowing reveals the following features: (i) when the microtubule outside is exposed the surface relief is dominated by the bound motor domains. Monomeric motor constructs generate a strong $8 \mathrm{~nm}$ periodicity, corresponding to the binding of one motor domain per $\alpha$ $\beta$-tubulin heterodimer. This surface periodic ity largely disappears when dimeric kinesin motor domains are used for decoration, even though it is still visible in negatively stained or frozen hydrated specimens. This could be explained by disorder in the binding of the second (loosely tethered) kinesin head, and/or disorder in the coiled-coil tail. (ii) Both surfaces of undecorated sheets or microtubules, as well as the inner surface of decorated sheets, reveal a strong $4 \mathrm{~nm}$ repeat (due to the periodicity of tubulin monomers) and a weak $8 \mathrm{~nm}$ repeat (due to slight differences between $\alpha$ - and $\beta$-tubulin). The differences between $\alpha$ - and $\beta$-tubulin on the inner surface are stronger than expected from cryo-electron microscopy of unstained microtubules, indicating the existence of tubulin sub- domain-specific surface properties that reflect the surface corrugation and hence metal deposition during evaporation. The $16 \mathrm{~nm}$ periodicity visible in some negatively stained specimens (caused by the pairing of cooperatively bound kinesin dimers) is not detected by surface shadowing.

Key words: Kinesin / M icrotubule sheets /

Surface topography / Unidirectional metal shadowing.

\section{Introduction}

Structural aspects of the interactions between kinesin and microtubules have been investigated by several authors using negative stain and cryo-electron microscopy and 3-D image analysis (for recent reviews see Amos and Hirose, 1997; Mandelkow and Hoenger, 1999; Vale and Milligan, 2000). The availability of atomic resolution X-ray structures of monomeric and dimeric kinesin (Kull et al., 1996; Sack et al., 1997; Kozielski et al., 1997) and ncd (Sablin et al., 1996, 1998) in combination with the near-atomic resolution EM-structure of zinc-induced tubulin sheets (Nogales et al., 1998) now provide detailed pictures of the microtubule structure (Nogales et al., 1999) and of the kinesin-tubulin complex (Hoenger et al., 2000; Kikkawa et al., 2000). There is now a general consensus on the tubulin interaction and binding geometry of monomeric kinesin and ncd (Hirose et al., 1995; Hoenger et al., 1995; Kikkawa et al., 1995; Arnal et al. 1996; Hoenger and Milligan, 1997). However, the microtubule binding properties of dimeric motor constructs are more complicated to investigate than the ones from monomers. Recent cryo-EM and scanning transmission electron microscopy (STEM)-based mass determinations (Müller et al., 1992) demonstrated the complexity of dimeric kinesin interaction with microtubules (Thormählen et al., 1998; Hoenger et al. 2000). While there is agreement on the arrangement of dimeric ncd (a retrograde kinesin-like motor) on the microtubule surface (S osa et al., 1997; Hirose et al., 1998), divergent interpretations have been given for the interaction of dimeric kinesin with microtubules (Arnal et al., 1996; Hirose et al., 1996, 1999; Hoenger et al., 1998, 2000). This situation has prompted us to study motor-microtubule complexes using complementary structural methods.

The tubulin 'sheets' (in the definition used here) are opened-up walls of microtubules and therefore exhibit nearly the same axial and lateral contacts between tubulin subunits as intact microtubules (Erickson, 1974), in contrast to the zinc-induced tubulin sheets which exhibit different lateral contacts between protofilaments (Amos and Baker, 1979; Tamm et al., 1979)and which have been used 
for the crystallographic determination of tubulin structure (Nogales et al., 1998). Tubulin sheets have been used previously for studying the arrangement of tubulin monomers (C repeau et al., 1977), the relationship between associated microtubule walls (Mandelkow and Mandelkow, 1979), and the relationship between open microtubule walls polymerized onto microtubules. They have as well been an important tool for determining microtubule polarity in cells (Heidemann and Mclntosh, 1980). The sheets have also been used to investigate the 3-D binding geometry of monomeric ncd and kinesin (Hoenger et al., 1995; Hoenger and Milligan, 1997) with negative stain electron microscopy and tilt-series 3-D reconstruction. Tubulin sheets are easy to handle with EM techniques that involve preadsorbtion to a carbon film (such as negative staining) because this causes them to flatten out and makes them suitable for image reconstruction, but they are not convenient for cryo-EM of frozen-hydrated samples because they retain their intrinsic cylindrical curvature (Mandelkow et al., 1991; Chrétien et al., 1995).

We applied freeze-drying and unidirectional tantalum/ tungsten ( $\mathrm{Ta} / \mathrm{W}$ ) shadowing to tubulin sheets decorated with monomeric and dimeric kinesin constructs to further investigate the binding properties of kinesin by using a method that allows to directly observe the surface topography with no need for computational data averaging and merging. This is made possible by the high signal/noise ratio of unidirectional shadowing and its explicit limitation of contrast enhancement to surface-exposed structural features. In comparison to this, the contrast contribution from
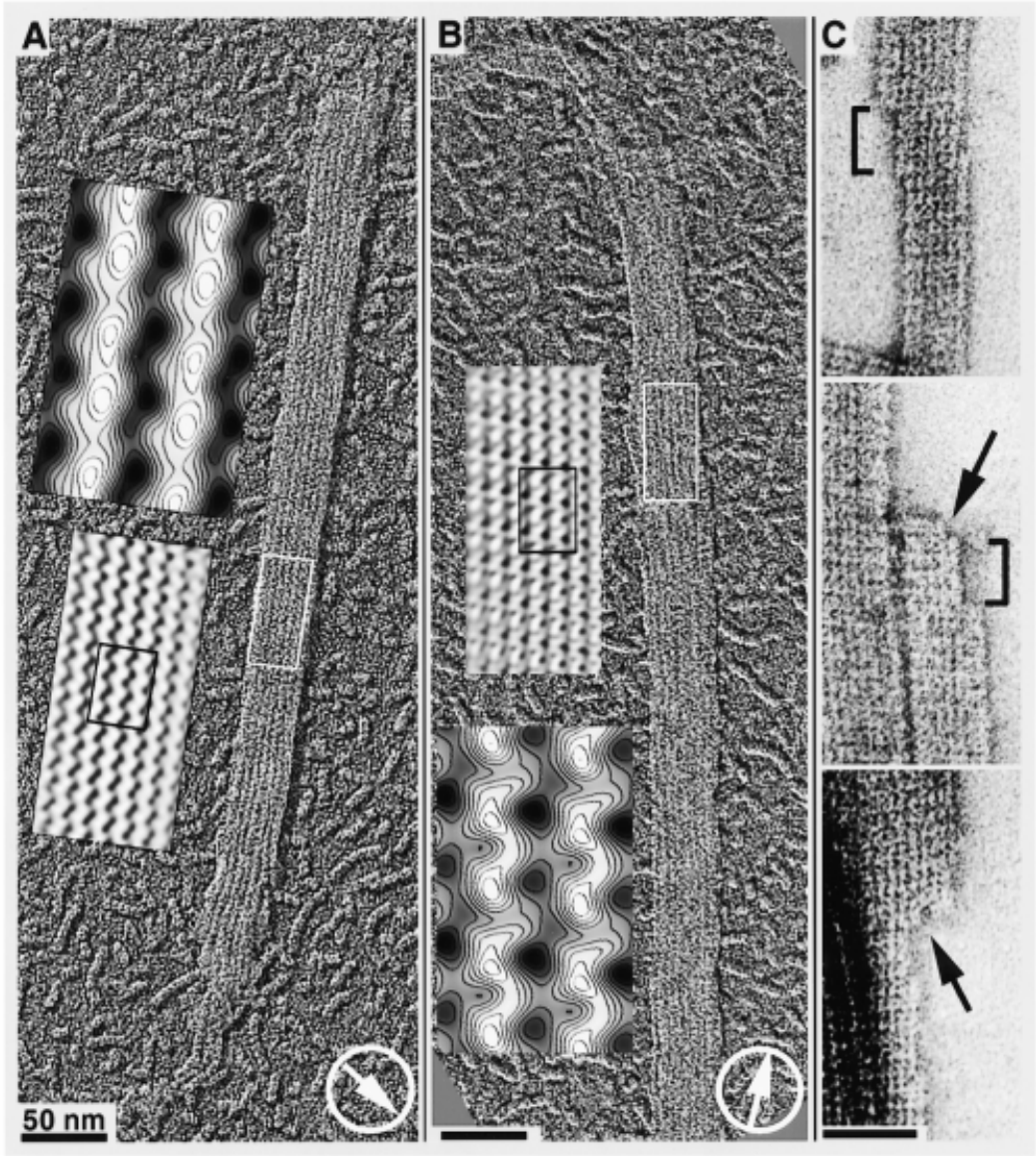

Fig. 1 Freeze-Drying and Unidirectional Surface Shadowing of Intact Microtubules.

A thin layer of Ta/W $(\sim 0.5 \mathrm{~nm})$ is applied to the surface of microtubules under an elevation angle of 45 degrees and in the azimuthal direction as indicated by the circled arrows, as is in all following figures. These preparations yield images, which show specifically the surface topography of the objects under investigation. Due to the intrinsic flexibility of lateral protofilament contacts in microtubules the shadowing procedure causes flattening so that the top surface of the microtubule collapses onto the bottom surface. Unlike for in the presence of motor proteins or other MAPs, which protect the integrity of microtubules, there is a strong tendency to depolymerize. The oligomeric structures in the background are multiples of tubulin dimers $(8 \mathrm{~nm}$ length) which break off from the sheets as illustrated in (C). Alternative shadowing directions reveal slightly different information. Shadowing in a direction roughly perpendicular to the protofilament axis (A) reveals the wavy, rim-like outer surface of protofilaments with large grooves in-between protofilaments (A). Insets show a Fourier-filtered area and a contour plot of an average of approx. 400 crystallographic unit cells in which $\alpha$-and $\beta$-tubulin have very similar shapes. A somewhat different appearance is obtained when the shadowing direction is pointing along the protofilament axis (B). Here the distinction between $\alpha$ - and $\beta$-tubulin becomes more obvious and allows outlining individual dimers (see Fourier-filtered area and contour plot). (C) Negatively stained tubulin sheets illustrating that protofilaments break off in multiples of tubulin dimers. The brackets in the top and in the middle panel indicate a gap of 4 dimers $(32 \mathrm{~nm})$. The arrows point to gaps of single dimers $(8 \mathrm{~nm})$. Scalebars $=50 \mathrm{~nm}$, axial repeats in insets $=8 \mathrm{~nm}$, lateral protofilament spacing $=5 \mathrm{~nm}$. 
non-surface exposed features is negligible. The appearance of a 'shadow' is caused by the directed metal vapor applied under a fixed azimuthal (indicated by circled arrows in every figure) and elevation angle (here kept constant at 45 degrees). This reveals images with a quasi-3-D appearance relative to surface modulations, somewhat comparable to an aerial photograph at sunset. Applied to regular, $2-D$ crystalline arrays this technique allows to directly observe surface features to $3-4 \mathrm{~nm}$. The resolution may be easily pushed below $1 \mathrm{~nm}$ on 2-D crystalline arrays using computational averaging (e.g. Walz et al. 1996). Here we took advantage of a freeze-drying/metal shadowing unit (Midilab; Gross et al., 1990) which allows transferring shadowed specimens directly into the specimen chamber of a Philips CM 12 cryo-electron microscope. By using this setup we could apply Ta/W shadowing without a stabilizing carbon coat (C-backing) obscuring fine structural details. In standard shadowing approaches a C-backing layer is necessary to minimize post-shadowing artifacts (rehydration, metal oxidation etc; Walz et al., 1996) during the exposure of the specimen to air and warming to ambient temperature.

It could be demonstrated by this work that surface shadowing constitutes a very suitable method to directly image the intrinsic disorder of randomly (double-) bound dimeric motor head constructs on the surface of 2-D crystalline arrays of tubulin sheets. As expected from previous results, monomeric kinesin motor heads bind to sheets in a regular B-lattice type pattern (Amos and Klug, 1974) revealing a strong $8 \mathrm{~nm}$ periodicity. Dimeric kinesins, however, revealed a significantly less ordered surface caused by their binding characteristics as predicted by Hoenger et al. (2000). Interestingly, the sheet surface corresponding to the microtubule inner side revealed surprisingly strong differences between the $\alpha$ - and $\beta$-tubulin units, similar to earlier observations made on negatively stained samples (Hoenger et al., 1995; Hoenger and Milligan, 1997).

\section{Results}

\section{Surface Features of Undecorated Mic rotubules and Tubulin Sheets}

The surface topography of microtubules reveals in a very direct way several structural features that have been found previously in 3-D reconstructions from negatively stained tubulin sheets (Crepeau et al., 1978; Hoenger et al., 1995), zinc-induced sheets (Amos and Baker, 1979), and frozen hydrated intact microtubules (e.g. Sosa et al., 1997; Hoenger et al., 1998). The outer rims of protofilaments (facing the outer microtubule surface) form a windy path which is best seen on pictures where the azimuth of shadowing direction lies roughly perpendicular to the protofilament axis (Figure 1A). Only a few minor topography modulations along the protofilament axis are visible. These modulations are more pronounced on images with a shadow azimuth pointing approximately along the protofilament direction (Figure 1B). These images also reveal more distinct features of the individual tubulin monomers. Unfortunately it is not possible on these pictures to clearly assign $\alpha$ - and $\beta$-tubulin to the 2-D averages. Even in the presence of taxol, EM-preparations of plain tubulin sheets, and in particular cryo-preparations such as freeze-drying and embedding in vitreous ice, are usually accompanied by a large amounts of free tubulin oligomers, which show up very clearly with this technique (see background in Figure 1A, B). This fragmentation is much less visible when microtubules are decorated with motor proteins or other MAPs, demonstrating the stabilizing effect of these associated proteins (see Figure 2). The (negatively stained) images of Figure $1 \mathrm{C}$ illustrate that tubulin is lost from the sheets in multiples of $\alpha \beta$-tubulin dimers (Figure $1 C$, brackets) or sometimes even as single dimers (Figure 1C, arrows). Microtubules, which undergo a freeze-drying process, do not maintain their tubular structure but always appear as flattened double-layered sheets (Figure 1A, B), indicating the flexible character of lateral protofilament contacts. Without this intrinsic flexibility the formation of flat sheets would probably not be possible at all.

\section{Surface Shadowing of Tubulin Sheets Decorated with Monomeric Kinesin Heads}

Decoration of tubulin sheets with monomeric kinesin head domains lacking the coiled-coil dimerization domain (nK343) allows a direct determination of inner and outer microtubule surface to a resolution of better than $4 \mathrm{~nm}$ (judged from the clear visibility of axial $4 \mathrm{~nm}$ repeats on the inner surface of sheets) without any need for computational averaging. Inner and outer surfaces of tubulin sheets decorated with monomeric kinesin heads are illustrated in Figure 2. The motor decoration strongly enhances the B-lattice type pattern (Amos and Klug, 1974; Song and Mandelkow, 1993) and the axial $8 \mathrm{~nm}$ repeats of individual $\alpha \beta$-tubulin dimers (Figure $2 \mathrm{~A}$, 'outer surface'). Inner microtubule surfaces, on the other hand, reveal the finer $4 \mathrm{~nm}$ repeat of the individual tubulin monomers, which are rather similar at that resolution (F igure 2A, 'inner surface'). The assignment of inner and outer surface is particularly obvious from the flattened 'corkscrew' structure of Figure 2B where the sheet flips over in alternating orientations, and the two sides of the tripartite composite sheet folded over in Figure $2 \mathrm{C}$. Tubulin sheets have a strong tendency to associate laterally into composite sheets (Mandelkow and Mandelkow, 1979; Figure 2A and C). This association may occurside-by-side with opposite orientation (Figures 2C, 3A and 3D), reminiscent of the lateral contacts between composite microtubule walls in cells (e.g. in flagellar doublet or triplet microtubules or 'S-shapes'). After flattening, decoration, and shadowing the alternating orientations become visible as alternating repeat patterns (outside surface dominated by $8 \mathrm{~nm}$ repeat and lattice lines inclined up and to the left in the direction of the left-handed helix of microtubules; inside 


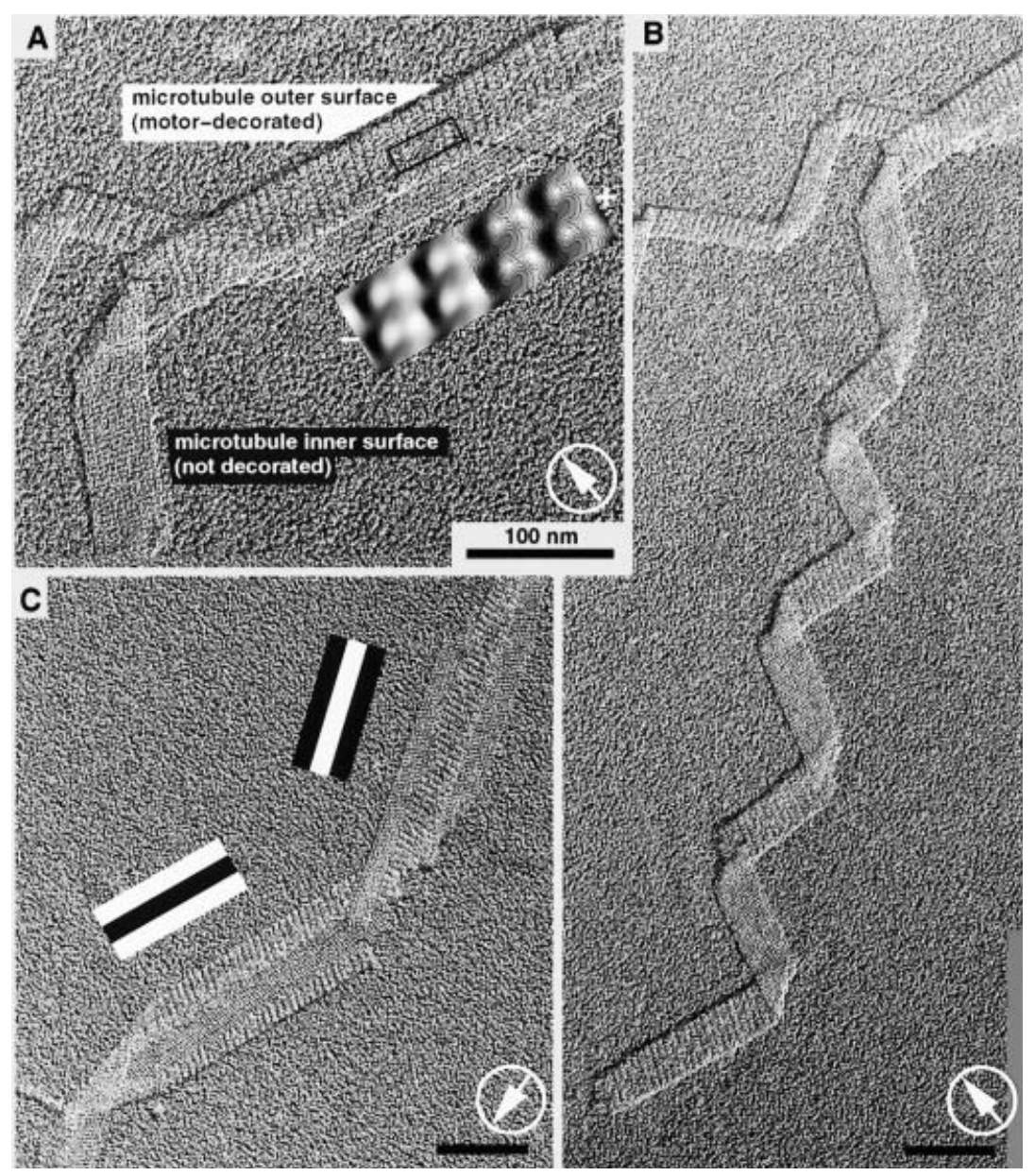

Fig. 2 Surface-Shadowed Tubulin Sheets Decorated with Monomeric Motor Domains (nK343) Clearly Reveal the Difference between Inner and Outer Microtubule Surfaces.

(A) A strong axial $8 \mathrm{~nm}$ repeat appears on sheets when the outer, motor-decorated, surface is exposed. This regular pattern corresponds to the axial repeat of tubulin dimers with one motor domain on each dimer. The inner surface exhibits a clearly different pattern corresponding to the axial elongation of approx. $4 \mathrm{~nm}$ of individual tubulin monomers. When a coiled sheet becomes flattened on the grid (B) the alternating domains have opposite orientations and therefore distinct repeat patterns ( $4 \mathrm{~nm}$ for exposed inside surface, $8 \mathrm{~nm}$ for exposed outside surface). Tubulin sheets have a tendency to associate laterally in several conformations. They frequently associate along the openly exposed lateral protofilament contacts $(C)$, which are covered in intact microtubules. This leads to composite sheets with alternating orientations and repeat patterns. The inset in A shows a crystallographic average of the outer surface (boxed area). Scalebars $=100 \mathrm{~nm}$.

surface dominated by $4 \mathrm{~nm}$ repeat and lattice lines inclined up and to the right). Even when sheets are joined with opposite orientation, this leaves two choices for the relative polarities (same polarity, as in microtubules, or opposite polarity, as in zinc-sheets). The polarities can be deduced from the density distributions of the motor surface (see Figure 2A, 3B, E). Initial polarity determinations have been carried out on negatively stained composite sheets (Hoenger and Milligan, 1996).

The images also illustrate that the 'B-lattice' arrangement of tubulin in the microtubule wall is by far the predominant one, where tubulin dimers in adjacent protofilaments are nearly in register (with a small stagger of $\sim 0.9$ $\mathrm{nm}$, generating the $\mathrm{J}_{3}$-helix seen in the lower part of Figure $2 A)$. B oth the ' $B$ ' and ' $A$ ' lattice were originally thought to occur in flagellar doublet microtubules (with adjacent tubulin dimers roughly in register or roughly half-staggered; Amos and Klug, 1974). However, it appears that the
A lattice-interaction is restricted to the closure of a microtubule wall ('seam') and therefore rarely observed in opened-up sheets. Such seams are a structural peculiarity of most microtubules; they correspond to a lattice mismatch that interrupts the helical symmetry of the microtubule (Mandelkow et al. 1986; Kikkawa et al., 1994; Song and Mandelkow, 1993, 1995). One explanation may be that seams, and therefore A-type interactions, are much weaker than B-type contacts. Individual sheets may simply split apart along these contacts. On the other hand, tubulin sheets are believed to be a polymeric form which occurs mostly in the earlier stages of polymerization and are the precursor of closed microtubules (Erickson, 1974; Mandelkow et al., 1991; Chretien et al., 1995). In this case the lattice mismatch would only occur during the closing process into a microtubule, and therefore sheets formed under the conditions used here may never have seams (see also Chretien et al., 1995; Desai and Mitchison, 1997). 


\section{Inner and Outer Surface Topography of Tubulin Sheets Decorated with Monomeric Kinesin Domains}

Figure $3 \mathrm{~A}-\mathrm{F}$ shows the results from crystallographic averaging of surface features with respect to different shadowing azimuthal angles. Taken together, Figures $3 \mathrm{~A}-\mathrm{C}$ and Figures 3D-F reveal a good picture of the surface topography of the outer and inner surface of kinesin-decorated tubulin sheets, which compares well with existing 3-D data. The topographies of the outer surfaces show comparable features to 3-D reconstructions of frozen hydrated kinesin-microtubule complexes (e.g. see Hoenger et al., 1998,2000 ) and even allow an ind irect polarity determination. J udging by the accumulation of metal (which appears bright) the kinesin head appears clearly higher and slight-

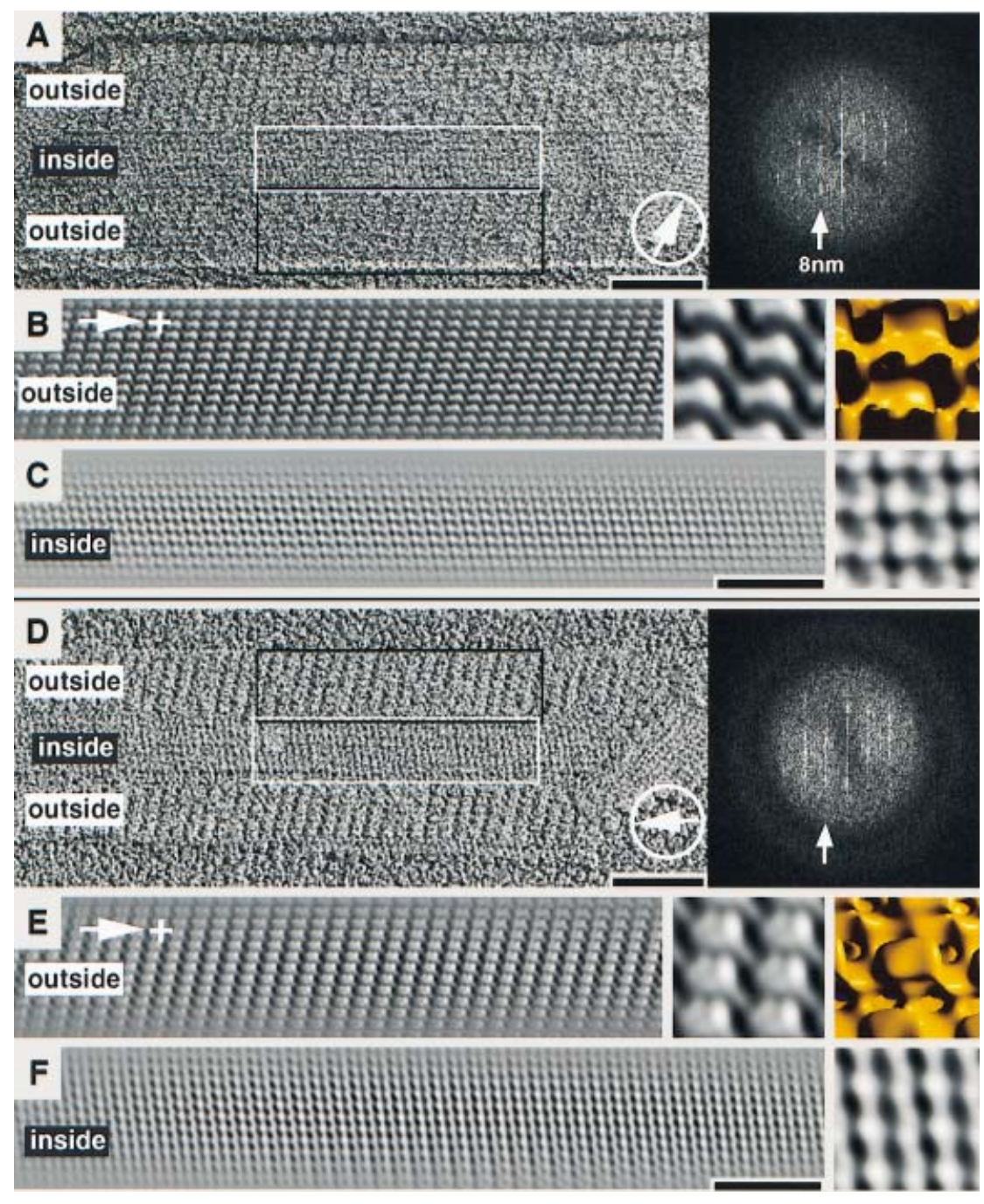

Fig. 3 Digital Image Analysis of Unidirectionally Shadowed Motor-Decorated Tubulin Sheets Reveals the Structural Details of the Outer Surface (Decorated) and Inner Surface (Non-Decorated) to a Resolution of Approx. $2 \mathrm{~nm}$.

Two shadowing directions at different azimuth angles are shown (circled arrows). (A) Image with a shadowing direction roughly perpendicular to the protofilament axis. Fourier-filtered and averaged areas from outer (B) and inner surface (C) illustrate the pronounced structural differences. On decorated outer surfaces the method of surface shadowing reveals predominantly the surface of the motors. The underlying outer tubulin surface is essentially hidden below. The apparent shape of the motor unit can be determined from the different shades of increasing metal depositions (bright). Accordingly the highest points and steepest slopes on the surface are the brightest areas in the averages, which in (B) and (E) point towards the plus end of the sheets (right, white arrow). This corresponds well with the shape of a kinesin head as determined from recent 3-D reconstructions and allows a polarity assignment as shown here, consistent with the preferential association of kinesin with $\beta$-tubulin (see Song and Mandelkow, 1993; Hoenger et al., 1998). The shadowing direction in panel A reveals the slightly tilted position of the motors with its highest elevation towards the plus-end of the protofilament. (D) Image of a specimen shadowed roughly along the protofilament axis $(D)$. The different shadowing directions reveal the shape of the attached kinesin motor domain. The lower and slightly flatter end points towards the minus-end ( $E$; for a detailed description of polarity determinations for 2-D and 3-D maps of microtubules see Hoenger and Milligan 1996). Interestingly we have never observed any lattice seams (A-lattice interactions) within a sheet. The axial shadowing direction (D) strongly emphasizes the axial $8 \mathrm{~nm}$ repeat corresponding to tubulin dimers arranged on a B-lattice, corresponding to a strong $8 \mathrm{~nm}$ lattice line in the diffraction patterns of both examples (see Song and Mandelkow, 1993). Note that averaged areas of inner surfaces also reveal significant differences between $\alpha$ - and $\beta$-tubulin (see also Figure 5 ). Scalebars $=50 \mathrm{~nm}$. 
ly narrower towards one side (the plus-end) of the protofilament and lower and flatter towards the other side (see Figure $3 B$ and $E$; Fourier-filtered arrays marked with a frame on Figure $3 A$ and $D$ ). There is a slight tilt of the head with respect to the protofilament axis, which is particularly clear with an axially directed evaporation angle as shown in Figure 3D and $E$. The intensities of averaged shadowgraphs (Figure 3, left inserts in $\mathrm{C}$ and $\mathrm{F}$ ) can be approximated using a 3-D volume from a helical reconstruction of kinesin-mic rotubule complexes with a computed illumination under the same azimuthal and elevation angles (Figure 3, right insets in $\mathrm{C}$ and F). Surface shadowing re-
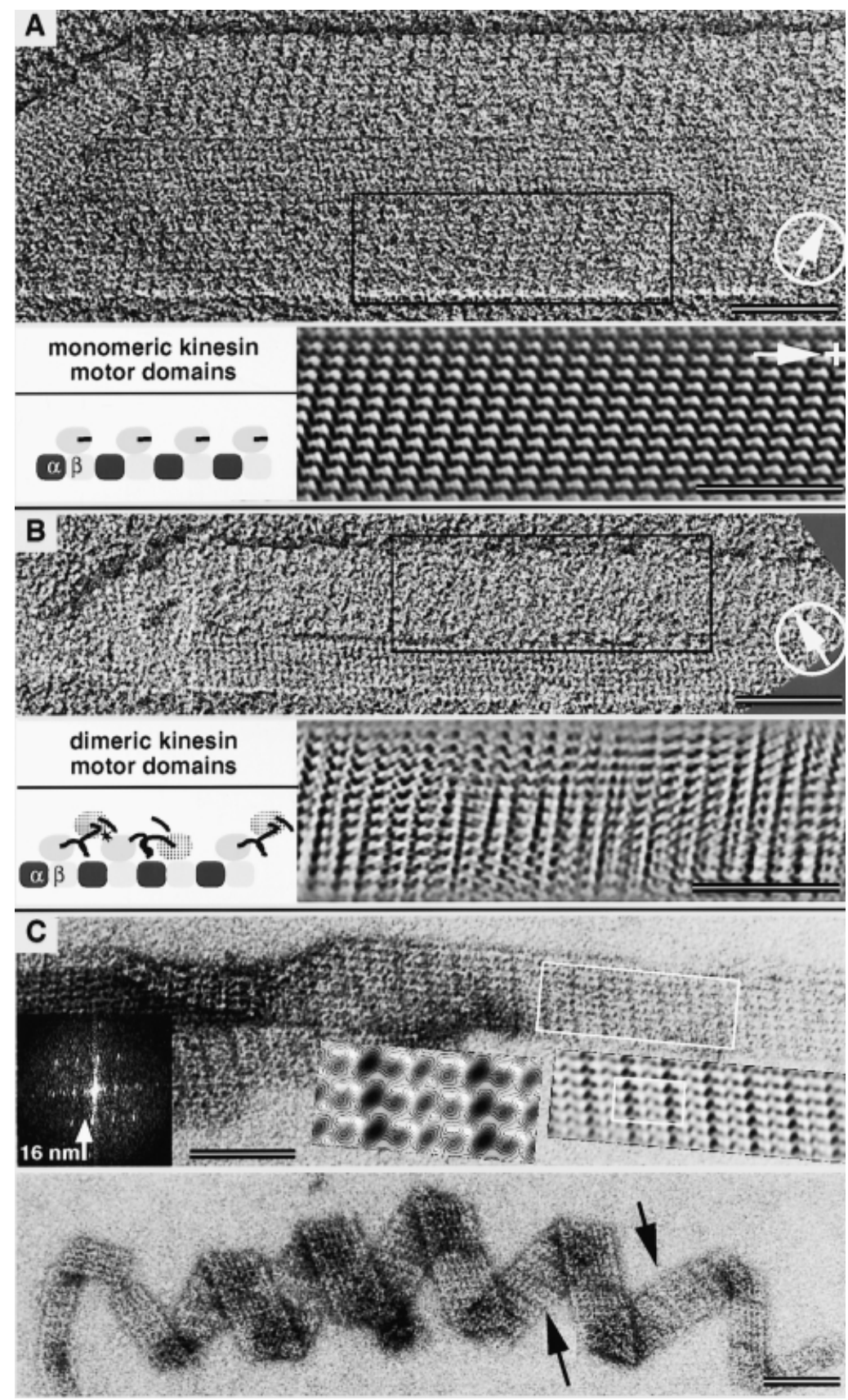

Fig. 4 Decoration of Tubulin Sheets with Monomeric Kinesin (nK343, A) and Dimeric Kinesin (rK379, B).

The pronounced $8 \mathrm{~nm}$ periodicity observed with monomeric kinesin (A, image and filtered image below) largely disappears in this sample of a sheet decorated with dimeric kinesin ( $B$, image and filtered image below). This is explained by a lack of ordered binding where a kinesin dimer may become attached to the tubulin lattice either with both heads bound to different $\beta$-tubulin monomers, or with only one head bound directly [the second one being loosely tethered, possibly in different spatial arrangements as illustrated on the sketches in (A) and (B); see Hoenger et al., 2000]. In exceptional situations the binding of dimeric kinesin (sK395) may occur in a cooperative manner, revealing an axial periodicity of $16 \mathrm{~nm}$ ( $C$, negatively stained specimen). This corresponds to the two heads of a kinesin dimer bound to two consecutive tubulin dimers (see Thormählen et al., 1998). However, so far we have not been able to reproduce these patterns for shadowed specimens. Scalebars $=50 \mathrm{~nm}$. 
veals mostly structural features from the bound motor domains. The underlying tubulin surface lattice is mostly hidden in the shadow of the motors (Figure 3B, E). Interestingly, $\alpha$ - and $\beta$-tubulin look remarkably different on the inner side of tubulin sheets, observed with both shadowing azimuths shown in Figure $3 \mathrm{C}$ and $\mathrm{F}$ (see also Figure 5). This particular finding is discussed in more detail below. This is unexpected since on cryo-images made in the absence of decoration the two tubulin subunit types are almost indistinguishable at this resolution (Nogales et al., 1999).

\section{Decoration with Dimeric Kinesin Constructs Generates a Disordered Surface Superimposed on an Ordered Binding Pattern}

In contrast to the decoration pattern of monomeric kinesin head domains (Figure 3, Figure 4A), the decoration with dimeric kinesin (rK379) does not generate a well-ordered periodic surface structure (Figure $4 \mathrm{~B}$ ). This is in striking contrast to negatively stained or frozen hydrated specimens where decoration by kinesin monomers and dimers leads to similar images (Hoenger et al., 1998; Thormählen etal., 1998). The disorder effect caused by dimers is strictly due to the bound kinesin, since the inside surface still shows the clear $4 \mathrm{~nm}$ periodicity typical of tubulin subunits (see lower part in Figure 4B). The distinct appearances must therefore be explained by the different imaging methods. The images obtained from both negatively stained and frozen hydrated particles represent a projection of the entire structure along the electron beam and are therefore dominated by the overall mass distribution, given by the tubulin and kinesin molecules, most of which are in a periodic arrangement. Since monomeric and dimeric kinesins bind such that each $\beta$-tubulin has one firmly attached motor domain, both yield similar images in a first approximation. On the other hand, unidirectional shadowing reflects the relief of the surface, independently of the underlying mass distribution. If the surface is irregular one would obtain 'blurred' pictures such as in Figure 4B.

What effects could cause the disorder? There are two likely possibilities, disorder in the 'loosely bound' head and disorder in the coiled-coil neck. As suggested previously (Hoenger etal., 2000), kinesin dimers can attach in at least two modes, either with both heads firmly attached to two different tubulin dimers, or with one head firmly attached and the second head loosely tethered. In a realistic situation (depending on the kinetics of decoration) one would expect a mixture of tubulin dimers with only one firmly bound kinesin head, with one firmly and one loosely bound head, and perhaps tubulin dimers without any bound kinesin. The projected structure would still be dominated by the tubulin lattice and the firmly anchored kinesin heads, but the surface would become highly irregular. This scenario would be consistent with the fractional stoichiometries observed by STEM (Thormählen et al., 1998; Hoenger et al., 2000). In addition, kinesin dimers are joined together by their helical tail, which under the condi- tions of shadowing is likely to collapse in random orientations, thus contributing further to an irregular surface.

In contrast to the situation described above, there are certain conditions (over which we do not have full control yet) where dimeric kinesin binds to mic rotubules in a highly cooperative and regular fashion, such that each head of a kinesin dimer binds to a $\beta$-tubulin subunit, and the bound kinesin dimers align in register. This generates a super-periodicity of $16 \mathrm{~nm}$ (Thormählen et al., 1998), as illustrated in

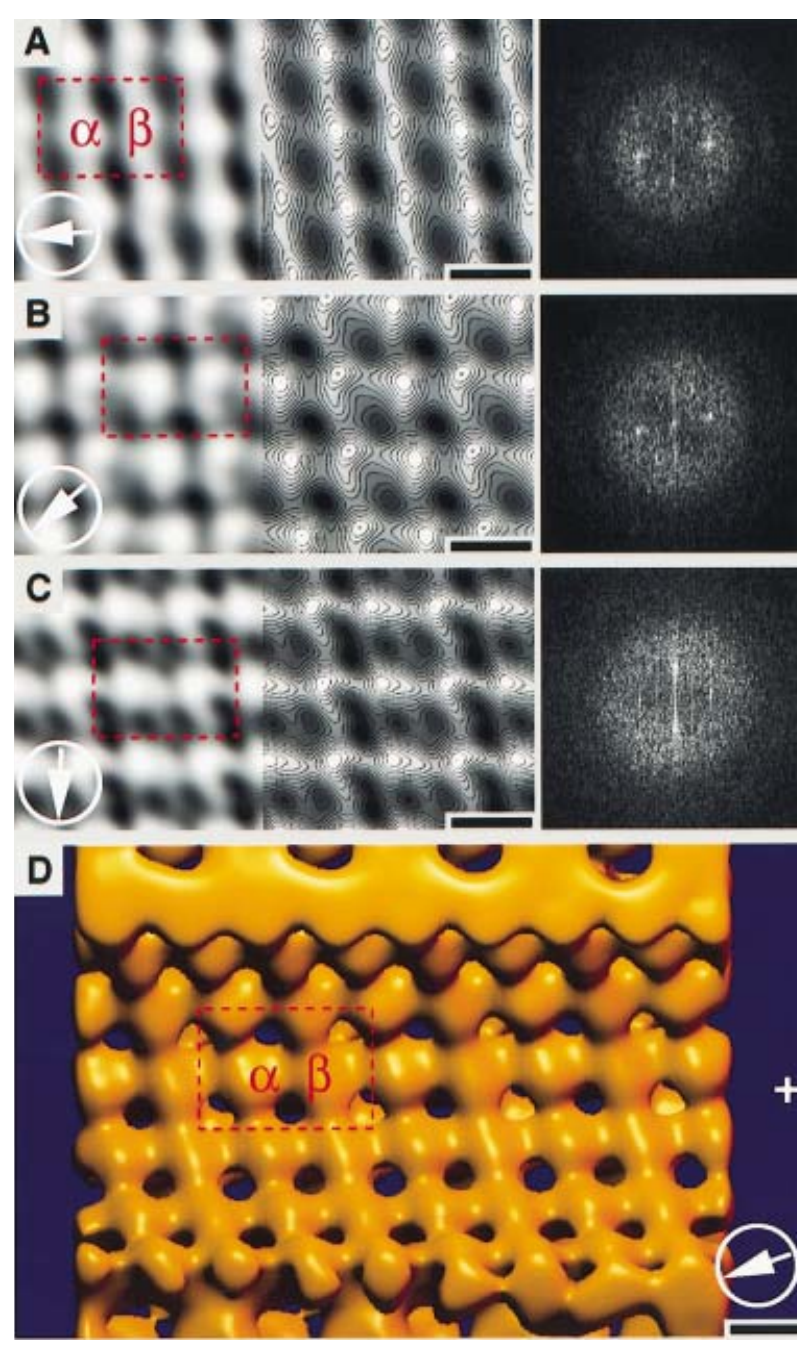

Fig. 5 Averages and Contour Plots of Unidirectionally Shadowed Motor-Decorated Microtubule Walls Whose Inner Surface Is Exposed and Therefore Not Decorated with Motor Domains. Structural differences between $\alpha$ - and $\beta$-tubulin are very apparent in all averages obtained from different shadowing azimuths as marked with arrows $(A-C)$. All diffraction patterns show a weak, but noticeable $8 \mathrm{~nm}$-lattice line. Unlike the outer surface of microtubules, which exhibit an almost continuous wavy rim in axial directions (see Figure 1), the inner surfaces show stronger lateral connections and deep laterally running grooves separating the inner protrusions of tubulin monomers (see Heuser, 1986). The shadowed specimen in $(A)$ agrees well with the inner surface topography of a helically reconstructed frozen-hydrated microtubule (D). Note that the rendered surface in the reconstruction (D) was illuminated along the protofilament axis, similar to the shadowing direction in (A). The $\alpha \beta$-tubulin dimer assignment in (A) is based on this comparison. Scale bars $=5 \mathrm{~nm}$. 
the negatively stained example of Figure $4 \mathrm{C}$, obtained by decoration with SK395. Thus far, this feature has not been detected in metal shadowed specimens, either because the conditions for cooperative binding were not met, or because the $16 \mathrm{~nm}$ periodicity - if it existed - was obliterated by the collapse of the protruding coiled-coil neck domains onto the surface in random orientations.

\section{The Surface Topographies \\ of the Inner Sides of Decorated and Plain Tubulin Sheets Reveal Signific ant Structural Differences between $\alpha$ - and $\beta$-Tubulin}

One of the unexpected findings of this work was the relatively pronounced structural difference between $\alpha$ - and $\beta$ tubulin on the inside surface of a microtubule wall. This was observed for a variety of azimuthal angles varying around 90 degrees (Figure 5A-C, circled arrows indicating the shadow azimuth). We have previously observed similar differences on tilt-series 3-D reconstructions of negatively stained tubulin sheets and sheets of tubulin-motor protein complexes (Hoenger et al., 1995; Hoenger and Milligan, 1997). A topograph obtained with an azimuth angle as shown in Figure $5 \mathrm{~A}$ compares well with the inner surface of a 3-D reconstruction from frozen-hydrated helical 15-protofilament microtubules shown with a simulated illumination under a comparable angle (Figure 5D). This allows an indirect assignment of the protofilament polarity and defines the $\alpha \beta$-tubulin dimer boundaries (compare Figure $5 A$ and $D$ ). Although the origin of this feature is currently unknown, the observation by two independent methods argues that the structural differences between $\alpha$ - and $\beta$-tubulin are genuine. The fact that even the finest resolved features show the directional nature of the shadowing process implies that decoration artifacts (non-uniform distribution of metal grains due to preferential nucleation on the surface) are negligible. The result is notable because model calculations based on the recently solved high-resolution structure of zinc-induced tubulin sheets (Nogales et al., 1998) would not imply that structural differences between the two units would be so obvious at the resolution of approx. $2 \mathrm{~nm}$. We therefore suggest that a microtubule either contains structural features, or is capable of conformational changes, which are not accounted for by the currently available structure of tubulin deduced from zinc-induced sheets. More specific investigations will be necessary to assess the nature of these observations in more detail.

\section{Discussion}

Although high-resolution structures of motor proteins and tubulin have been obtained over the past few years, many features of the interaction between microtubules and motors that generate movement are still unknown. This applies in particular to the structural aspects of microtubulemotor complexes. These complexes are generally not suitable for high resolution $X$-ray diffraction studies so that one has to rely on intermediate resolution electron imaging approaches, combined with image processing and reconstruction. Their advantage is that they can be applied in a number of ways, which yield distinct and complementary views of the structure. In recent years we have studied microtubule walls (sheets) without or with decoration by various recombinant kinesin constructs, using negatively stained or unstained frozen-hydrated samples which represent a reliable approximation to the native structure. In both cases one obtains projection views from which the surface topography can only be extracted indirectly by tilt series and image reconstruction (for a review see Mandelkow and Hoenger, 1999). In the present study we have addressed the surface structure directly, using unidirectional metal shadowing at the highest possible resolution in a specially designed apparatus (MIDILAB; Gross et al., 1990).

The results confirm some structural features anticipated by previous results, but they also reveal novel aspects of the decorated mic rotubule structure:

(i) Microtubules are flattened during the specimen preparation but otherwise retain the groove and ridge structure typical of the longitudinal protofilaments. In the absence of motors or other MAPs, even taxol-stabilized microtubules are labile, and protofilament fragments representing the breakdown products can clearly be recognized throughout the background (Figure 1 ). This means that in principle the method lends itself to time-resolved studies of processes such as assembly or motor binding. The disassembly occurs in multiples of the tubulin dimer, consistent with its role as the building block of microtubules.

(ii) There is a clear distinction between the inner and outer surface of decorated microtubules. The inner surface resembles that of a pure microtubule lattice and is dominated by the $4 \mathrm{~nm}$ repeat of tubulin monomers. Only the outer surface binds kinesin heads and therefore shows the $8 \mathrm{~nm}$ repeats typical of one strongly bound head per tubulin heterodimer (Figure 2).

(iii) Where the lattice is visible, it clearly reveals the $B$-lattice in which adjacent tubulin dimers are nearly in register. Seams where adjacent tubulin dimers are half-staggered (A-lattice interaction) are extremely rare, arguing that the $B$-lattice is the preferred mode for aggregation.

(iv) The surface features of bound kinesin heads compare well with those deduced from the frozen-hydrated specimens, indicating that shadowing yields a faithful representation of the native structure (Figure 3). The polarity of the bound kinesin can be identified and agrees with the model proposed earlier (Hoenger et al., 1998).

(v) The $16 \mathrm{~nm}$ super-period, due to cooperative binding of kinesin dimers, has been detected by negative staining but not by shadowing (Figure 4), nor on frozen-hydrated specimens, possibly because of the inherent disorder in the surface of bound kinesin dimers.

(vi) The low degree of order found with kinesin dimers is in strong contrast to the high order when monomers decorate the microtubule lattice. This is an unexpected find- 
ing; it suggests that the attachment of dimers can take different routes, with loosely tethered heads possibly accounting for the disorder, in combination with the collapse of the coiled-coil tails onto the surface. In this regard, the surface relief is distinctly different from the bulk structure obtained from image reconstruction of frozen-hydrated specimens (e.g. Hoenger et al., 2000).

(vii) The tubulin structure itself shows features of $8 \mathrm{~nm}$ periodicities, especially on the inside surface, which were not anticipated by previous experiments and are not explained by the current tubulin structure.

(viii) Finally, perhaps the greatest advantage of the method is its high inherent contrast, which allows interpretation at detail below $4 \mathrm{~nm}$ resolution without any image enhancement. This means that even non-periodic structural features can be studied. We therefore expect that the method will help to bridge the gap between structural and kinetic studies, and between the study of single particle interactions and large macromolecular assemblies.

\section{Materials and Methods}

\section{Expression of Monomeric and Dimeric Kinesin Constructs}

Kinesin constructs used here were Neurospora kinesin nK343 (monomeric; Figures 2, 3, 4A), (a kind gift of Dr. M. Schliwa and Dr. G. Woehlke, Muenchen), squid kinesin SK395 (dimeric; Figure 4C), and rat kinesin rK379 (dimeric; Figure 4B). Plasmids coding for rat kinesin constructs were cloned and expressed as described in Kozielski et al. (1997a). B riefly, to obtain pErk379 the BamHI-Saul-fragment of the rat kinesin gene (kindly provided by S. Brady, unpublished) was inserted into a $\mathrm{pET}$-3a vector modified to contain the same sites with a stop codon. The plasmids were expressed in E.coli BL21 (DE3) cells. Recombinant rK379 could be obtained in soluble form from the bacterial extracts. E. coli cells were grown in LB Medium and expression of kinesin was induced with $0.4 \mathrm{mM}$ IPTG at a cell density corresponding to $A 600=0.6-0.8$. Cells were harvested after 16 hours of induction at $25^{\circ} \mathrm{C}$, resuspended in lysis buffer ( $50 \mathrm{~mm}$ PIPES, pH 6.9, $60 \mathrm{~mm}$ $\mathrm{NaCl}, 1 \mathrm{~mm} \mathrm{MgCl} 2,0.5 \mathrm{~mm}$ EGTA, $2 \mathrm{~mm}$ DTT, $1 \mathrm{~mm}$ PMSF) and lysed with a French press. Purification was done by ion exchange chromatography on phosphocellulose and MonoQ followed by gel filtration on a G-200 Hiload 16/60 column (Pharmacia). Squid kinesin was expressed and purified according to Song and Mandelkow (1993).

\section{Motor Decoration of Tubulin Sheets}

Tubulin was prepared by phosphocellulose chromatography preceded by a MAP-depleting step as described (Mandelkow et al., 1985). Preparation of motor-decorated tubulin sheets was performed as described in Hoenger and Milligan (1997). B riefly, tubulin at $5 \mathrm{mg} / \mathrm{ml}$ was polymerized for 30 minutes at $37^{\circ} \mathrm{C}$ in $80 \mathrm{~mm}$ Pipes pH 6.8, $4 \mathrm{~mm} \mathrm{MgCl}_{2}$ (= BRB 80) supplemented with $10 \%$ DMSO, 2 mM GTP and the resulting microtubules and sheets were stabilized with $20 \mu \mathrm{M}$ taxol. Monomeric and dimeric kinesin was added to the polymerized sheet solution at a 2.5 -fold excess over potential binding sites (tubulin dimers). To one volume of a tubulin solution at $0.5 \mathrm{mg} / \mathrm{ml}$ we added one volume of a kinesin solution at a concentration of $1 \mathrm{mg} / \mathrm{ml}$. AMP-PNP was supplemented to a final concentration of $20 \mu \mathrm{M}$. The resulting mix was incubated for 3 - 4 min and then directly applied to glow-discharged carboncoated electron microscopy grids.

\section{Freeze-Drying and Metal Shadowing}

A few microlitres of a tubulin sheet solution (with or without complexed motors) at a concentration of approx. $0.5 \mathrm{mg} / \mathrm{ml}$ tubulin was applied to carbon-coated grids and adsorbed for $2-3 \mathrm{~min}$. Then the grids were washed twice in BRB80 and once in doubledistilled water, blotted to remove excess liquid and quick-frozen in liquid nitrogen. Frozen grids were then transferred to the socalled 'Midilab'. The Midilab is a unique design of the lab of $\mathrm{H}$. Gross and is essentially a freeze-drying/metal shadowing unit, which is mounted onto the column of a Philips-CM 12 electron microscope at the height of the specimen stage and allows for a direct vacuum cryo-transfer of specimens into the microscope onto a modified GATAN cryo-holder (Gross et al., 1990). In the Midilab samples were freeze dried for 2 hours at $180 \mathrm{~K}$ and a pressure of $<5 \times 10^{-7}$ mbar. Properly dried specimens were subsequently unidirectionally shadowed with a $0.5 \mathrm{~nm}$ thick layer of tantalum/ tungsten (Ta/W) at an elevation angle of 45 degrees. Unlike for regular preparations, which have to be transferred through air, no carbon backing is needed in this setup, which results in a significant increase of resolution and a higher signal/noise ratio. The contrast created by the metal coat is so high that the contrast component resulting from unstained material below the surface remains negligible.

\section{Image Analysis}

Images were recorded by a GATAN-794 Multiscan CCD camera (GATAN, Pleasanton, CA, USA) at an electron dose of $500-1000$ electrons $/ \mathrm{nm}^{2}$. Suitable areas for Fourier filtering and averaging were computationally unbend using the MRC-based SPECTRA platform (Schmid et al., 1993), Fourier filtered, and averaged using the MRC (Henderson et al., 1986) and SUPRIM packages (Schroeter and Bretaudiere, 1996). The Fourier filtered images and averages in Figure $4 \mathrm{~B}$ and $\mathrm{C}$ were obtained assuming lattice parameters with $16 \mathrm{~nm}$ axial repeats (corresponding to two consecutive tubulin dimers along the protofilament axis). All other averages assumed an $8 \mathrm{~nm}$ axial repeat, according to the length of one tubulin dimer. The lateral lattice parameters were always kept according to the lateral distance between two protofilaments $(=c a .5 \mathrm{~nm}$ ). Helical 3-D reconstructions as the one shown in Figure 5D have been obtained from frozen-hydrated specimen of helical 15-protofilament microtubules as described in Sosa et al. (1997) and Hoenger et al. (1998).

\section{Acknowledgements}

We thank J ens Müller and Manfred Thormählen (MPG, DESYHamburg) for the preparation of rat kinesin. This work was supported by a grant from the Swiss Science Foundation to A. Hoenger and H.Gross.

\section{References}

Amos, L., and Klug, A. (1974). Arrangement of subunits in flagellar microtubules. J. Cell Sci. 14, 523 - 549.

Amos, L.A., and Baker, T.S. (1979). The three-dimensional structure of tubulin protofilaments. Nature 279, $607-612$.

Amos, L.A., and Hirose, K. (1997). The structure of microtubulemotor complexes. Curr. Opin. Cell Biol. 9, 4 - 11.

Arnal, I., Metoz, F., DeBonis, S., and Wade, R.H. (1996). Three-dimensional structure of functional motor proteins on microtubules. Curr. Biol. 6, 1265 - 1270. 
Crepeau, R.H., McEwen, B., Dykes, G., and Edelstein, S.J . (1977). Structural studies on porcine brain tubulin in extended sheets. J. Mol. Biol. 116, $301-315$.

Crepeau, R.H., McEwen, B., and Edelstein, S.J . (1978). Differences in $\alpha$ and $\beta$ polypeptide chains of tubulin resolved by electron microscopy with image reconstruction. Proc. Natl. Acad. Sci. USA 75, 5006 - 5010.

Chrétien, D., Fuller, S.D., and Karsenti, E. (1995). Structure of growing microtubule ends: two-dimensional sheets close into tubes at variable rates. J . Cell Biol. 129, 1311 - 1328.

Desai, A., and Mitchison, T.J . (1997). Microtubule polymerization dynamics. Annu. Rev. Cell Dev. Biol. 13, 83 - 117.

Erickson, H.P.J . (1974). Microtubule surface lattice and subunit structure and observation on reassembly. J. Cell Biol. 60, 153 167.

Gross, H., Krusche, K., and Tittmann, P. (1990). Recent progress in high resolution shadowing for biological TEM. XIIth International Congress for Electron Microscopy, Seattle, USA, $510-511$.

Heidemann, S.R., and McIntosh, J.R. (1980). Visualization of the structural polarity of mic rotubules. Nature 286, $517-519$.

Henderson, R., Baldwin, J .M., Downing, K.H., Lepault, J ., and Zemlin, F. (1986). Structure of purple membrane from Halobacterium halobium: recording, measurement and evaluation of electron micrographs at $3.5 \AA$ resolution. Ultramicroscopy 19 , 147 - 178.

Heuser, J .E. (1986). Different structural states of a microtubule cross-linking molecule, captured by quick-freezing motile axostyles in protozoa. J . Cell Biol. 103, 2209 - 2227.

Hirose, K., Lockhart, A., Cross, R.A., and Amos, L.A. (1995). Nucleotide dependent angular change in kinesin motor domain bound to tubulin. Nature 376, $277-279$.

Hirose, K., Lockhart, A., Cross, R.A., and Amos, L.A. (1996). Three-dimensional cryoelectron microscopy of dimeric kinesin and ncd motor domains on microtubules. Proc. Natl. Acad. Sci. USA 93, 9539 - 9544.

Hirose, K., Cross, R.A., and Amos, L.A. (1998). Nucleotide-dependent structural changes in dimeric Ncd Molecules complexed to microtubules. J. Mol. Biol. 278, $389-400$.

Hirose, K., Löwe, J., Alonso, M., Cross, R.A., and Amos, L.A. (1999). Congruent docking of dimeric kinesin and nod into three-dimensional electron cryomicroscopy maps of microtubule-motor ADP complexes. Mol. Biol. Cell 10, 2063 - 2074.

Hoenger, A., Sablin, E.P., Vale, R.D., Fletterick, R.J ., and Milligan, R.A. (1995). Three-dimensional structure of a tubulin-motorprotein complex. Nature 376, $271-274$.

Hoenger, A., and Milligan, R.A. (1996). Polarity of 2-D and 3-D maps of tubulin sheets and motor decorated sheets. J. Mol. Biol. 263, 114 - 119.

Hoenger, A., and Milligan, R. A. (1997). Motor domains of kinesin and ncd interact with microtubule protofilaments with the same binding geometry. J . Mol. Biol. 265, $553-564$.

Hoenger, A., Sack, S., Thormählen, M., Marx, A., Muller, J ., Gross, H., and Mandelkow, E. (1998). Image reconstruction of microtubules decorated with monomeric and dimeric kinesins: comparison with X-ray structure and implications for motility. J . Cell Biol. 141, $419-430$.

Hoenger, A., Thormählen, M., Diaz-Avalos, R., Doerhoefer, M., Goldie, K., Muller, J ., and Mandelkow, E. (2000). A new look at the microtubule binding patterns of dimeric kinesins. J. Mol. Biol. 297, 1087 - 1103.

Kikkawa, M., Ishikawa, T., Nakata, T., Wakabayashi, T., and Hirokawa, N. (1994). Direct visualization of the mic rotubule lattice seam both in vitro and in vivo. J. Cell Biol. 127, 1965 - 1971.

Kikkawa, M., Ishikawa, T., Wakabayashi, T., and Hirokawa, N. (1995). Three-dimensional structure of the kinesin head-micro- tubule complex. Nature 376, 274 - 277.

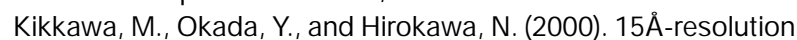
model of the monomeric kinesin motor KIF1A. Cell 100, 241 252.

Kozielski, F., Sack, S., Marx, A., Thormählen, M., Schönbrunn, E., Biou, V., Thompson, A., Mandelkow, E.-M., and Mandelkow, E. (1997a). The crystal structure of dimeric kinesin and implications for microtubule-dependent motility. Cell 91, 985 - 994.

Kozielski, F., Schönbrunn, E., Sack, S., Müller, J ., B rady, S.T., and Mandelkow, E. (1997b). Crystallization and preliminary X-ray analysis of the single-headed and double-headed motor protein kinesin. J. Struct. Biol. 119, 28 - 34.

Kull, F.J ., Sablin, E., Lau, P., Fletterick, R., and Vale, R. (1996). Crystal structure of the kinesin motor domain reveals a structural similarity to myosin. Nature 380, $550-554$.

Mandelkow, E.-M., and Mandelkow, E. (1979). J unctions between microtubule walls. J. Mol. Biol. 129, 135 - 148.

Mandelkow, E.-M., Herrmann, M., and Rühl, U. (1985). Tubulin domains probed by limited proteolysis and subunit-specific antibodies. J. Mol. Biol. 185, 311 - 327.

Mandelkow, E.-M., Schultheiss, R., Rapp, R., Müller, M., and Mandelkow, E. (1986). On the surface lattice of microtubules: helix starts, protofilament number, seam and handedness. J . Cell Biol. 102, 1067 - 1073.

Mandelkow, E.-M., Mandelkow, E., and Milligan, R.A. (1991). Microtubule dynamics and microtubule caps: a time-resolved cryo-electron microscopy study. J . Cell Biol. 114, 977 - 991.

Mandelkow, E., and Hoenger, A. (1999). Structures of kinesin and kinesin-microtubule interaction. Curr. Opin. Cell Biol. 11, 34 44.

Müller, S.A., Goldie, K.N., Bürki, R., Häring, R., and Engel, A. (1992). Factors influencing the precision of quantitative scanning transmission electron microscopy. Ultramicroscopy 46 , $317-334$

Nogales, E., Wolf, S., and Downing, K.H. (1998). Structure of the $\alpha \beta$-tubulin dimer by electron crystallography. Nature 391, $199-203$.

Nogales, E., Whittaker, M., Milligan, R.A., and Downing, K.H. (1999). High-resolution model of the microtubule. Cell 96, $79-88$.

Sablin, E.P., Kull, F.J ., Cooke, R., Vale, R.D., and Fletterick, R.J . (1996). Crystal structure of the motor domain of the kinesin-related motor ncd. Nature 380, 555 - 559.

Sablin, E.P., Case, R.B., Dai, S.C., Hart, C.L., Ruby, A., Vale, R.D., and Fletterick, R.J. (1998). Direction determination in the minus-end-directed kinesin motor Ncd. Nature 395, $813-$ 816.

Sack, S., Müller, J ., Marx, A., Thormählen, M., Mandelkow, E.-M., Brady, S.T., and Mandelkow, E. (1997). X-ray structure of motor and neck domains from rat brain kinesin. Biochemistry 36 , 16155 - 16165.

Schmid, M.F., Dargahi, R., and Tam, M.W. (1993). SPECTRA: a system for processing electron images of crystals. Ultramicroscopy 48, 251 - 264.

Schroeter, J ., and Bretaudiere, J.-P. (1996). SUPRIM: Easily modified image processing software. J . Struct. Biol. 116, 131 - 137.

Song, Y.H., and Mandelkow, E. (1993). Recombinant kinesin motor domain binds to beta-tubulin and decorates microtubules with a B surface lattice. Proc. Natl. Acad. Sci. USA 90, 1671 1675.

Song, Y.-H., and Mandelkow, E. (1995). The anatomy of flagellar microtubules: polarity, seam, junctions and lattice. J. Cell Biol. $128,81-94$.

Sosa, H., Dias, D.P., Hoenger, A., Whittaker, M., Wilson-Kubalek, E., Sablin, E., Fletterick, R.J., Vale, R.D., and Milligan, R.A. (1997). A model for the microtubule-Ncd motor protein com- 
plex obtained by cryo-electron microscopy and image analysis. Cell 90, $217-224$.

Tamm, L.K., Crepeau, R.H., and Edelstein, S.J . (1979). Threedimensional reconstruction of tubulin in zinc-induced sheets. J. Mol. Biol. 130, $473-492$.

Thormählen, M., Marx, A., Müller, S.A., Song, Y.-H., Mandelkow, E.-M., Aebi, U., and Mandelkow, E. (1998). Interaction of monomeric and dimeric kinesin with microtubules. J . Mol. Biol. 275, $795-809$.
Vale, R.D., and Milligan, R.A. (2000). The way things move: looking under the hood of molecular motor proteins. Science 288 , $88-95$.

Walz, T., Tittmann, P., Fuchs, K.H., Mueller, D., Smith, B.L., Agre, P., Gross, H., and Engel, A. (1996). Surface topographies at subnanometer-resolution reveal asymmetry and sidedness of aquaporin-1. J. Mol. Biol. 264, 907 - 918.

Received J une 20, 2000; accepted J uly 14, 2000 